\title{
A tool for modelling telluric spectra
}

\author{
Alain Smette ${ }^{1}$, Hugues Sana ${ }^{1,2}$ and Hannes Horst ${ }^{1,3}$ \\ ${ }^{1}$ ESO, Alonso de Cordova 3107, Casilla 19001, Vitacura Santiago, Chile \\ email: asmette@eso.org \\ ${ }^{2}$ Universiteit van Amsterdam, Sterrenkundig Instituut 'Anton Pannekoek', \\ Postbus 94249, - NL-1090 GE Amsterdam, The Netherlands email: hsana@eso.org \\ ${ }^{3}$ now at EOS GmbH - Electro Optical Systems, Niederlassung Chemnitz, \\ Annaberger Strasse 240, D-09125 Chemnitz, Germany email: Hannes.Horst@eos.info
}

\section{Introduction}

Accurate synthetic telluric spectra are required for efficient use of telescope time, in particular, with large telescopes and high-resolution NIR spectroscopy: (i) In the preparation of observations, are the telluric features at the same wavelength as spectroscopic features of scientific interest? Since water vapor is the molecule whose abundance varies most in the atmosphere, what values of precipitable water vapor are suitable to carry out successful observations? Are the observations of a telluric star required? Or better, can telluric features in the science spectrum be accurately represented by an appropriate synthetic spectrum? This point is also very important in the planning of telescope time, as observations of a telluric star may sometimes take longer than the one of the science target. (ii) In the analysis of the observations, how do telluric lines affect the scientifically interesting features in the observed spectrum? Is it possible to recover the useful information when telluric star observations could not be obtained, do not have sufficient SNR, or suffer from a significant change in instrumental or observing conditions?

A number of authors have already proposed synthetic telluric spectra to help analyze spectral features, e.g, Bailey, Simpson \& Crisp (2007), but did not include the possibility to change the temperature and pressure profile and the column densities of the relevant molecules in the Earth atmosphere. In the following, we describe molecfit, a tool to provide accurate synthetic spectra for use in Exposure Time Calculators, and in determining column densities of relevant molecules in the atmosphere based on either sky emission spectra in the thermal infra-red or in transmission spectra at any wavelength long-wards of $400 \mathrm{~nm}$. In its most complex form, it accurately models the telluric lines affecting a given spectrum. A systematic check of the accuracy of molecfit over the NIR range covered by CRIRES (Käufl et al. 2004) is being carried out.

\section{Requirements}

A tool aiming at accurately modelling telluric spectra requires: (a) an accurate radiative transfer code, able to take into account the most important aspects of the radiative transfer physics taking place in the Earth atmosphere; we choose RFM, the Reference Forward Model (Dudhia 2008); (b) an accurate and complete database of molecular parameters, such as HITRAN (Rothman et al. 2009); (c) an atmospheric profile - appropriate for the time of observations that accurately describes the change of temperature, pressure and abundance of the different molecules with altitude: The molecular abundance profiles are provided by RFM. Temperature and pressure profiles adapted for the time and place of the observations can be obtained through http://ready.arl.noaa.gov/READYamet.php.

Adapting the code to reproduce observed telluric lines requires additional capabilities: (d) the column densities of the different molecules involved have to be adjusted to match those at the time of the observations; (e) the wavelength calibration provided by a data reduction tool is usually not accurate enough and needs to be also re-determined based on the telluric lines themselves; $(f)$ the spectral resolution may depend on the accurate light profile at the entrance slit; in the case of CRIRES, it may fluctuate with the adaptive optics profile; its value must therefore also be adjusted by the code; $(g)$ the response curve of the whole system (instrument, 

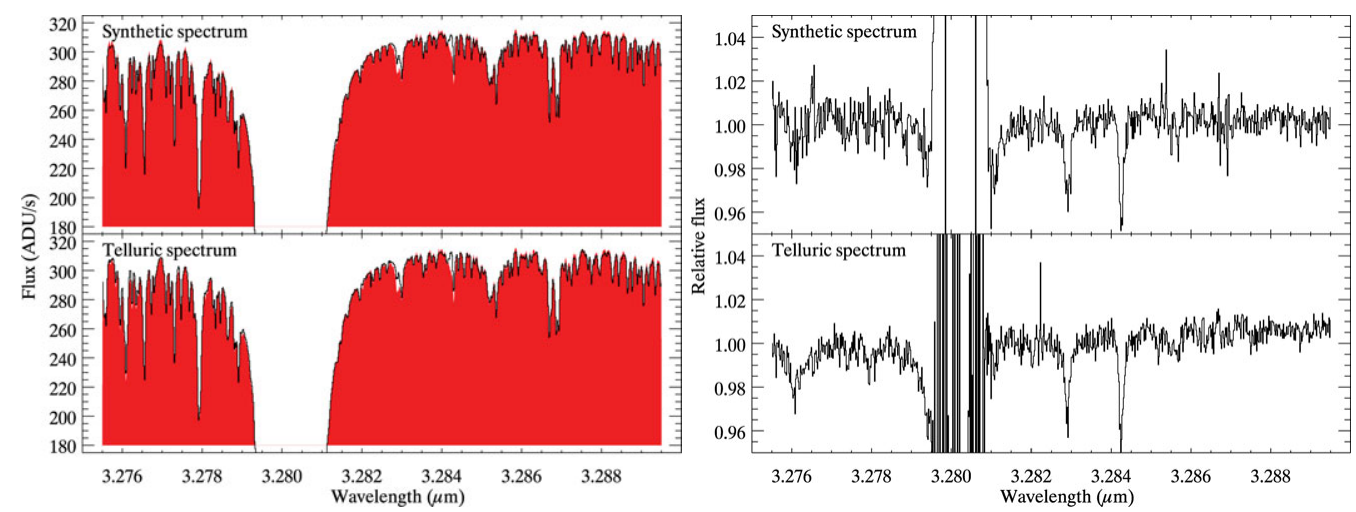

Figure 1. Comparison of correction between a telluric and a synthetic spectrum. (Left:) shaded (red in the online version): extract of a CRIRES spectrum of a star showing 2 intrinsic absorption features; (solid line, top:) synthetic spectrum obtained with molecfit; (solid line, bottom:) telluric star spectrum taken with an airmass difference smaller than 0.01. (Right:) Relative difference between the science spectrum and the synthetic spectrum (top) and a telluric spectrum (bottom).

telescope, atmosphere) needs to be adjusted for accurate corrections; $(h)$ some data points, either fixed (detector defects) or wavelength dependent (known stellar features) must not be used by the fitting algorithm.

\section{Applications}

This tool is already used to create the sky spectra (both emission and absorption) used by the CRIRES and E-ELT Exposure Time Calculators and to measure the amount of precipitable water vapor with CRIRES and VISIR (Lagage et al. 2004) in real time (cf. : http://www. eso.org/sci/facilities/paranal/sciops/CALISTA/pwv/data.html). It is currently being adapted to measure systematically the column density of other molecules, such as $\mathrm{CO}_{2}, \mathrm{CO}, \mathrm{O}_{3}, \mathrm{CH} 4$.

As shown in Fig. 1, the quality of the correction by the synthetic spectrum can be very similar to (or better than) the one by the telluric spectrum.

\section{References}

Bailey, J., Simpson, A., \& Crisp, D. 2007, PASP 119, 228

Dudhia, A. 2008 http://www.atm.ox.ac.uk/RFM/index.html

Käufl et al., 2004, SPIE 5492, 1218

Lagage, P. O. et al. 2004, The Messenger 117, 12

Rothman, L. S., et al., 2009, JQSRT 110, 9 